\title{
Isolation and characterization of oleaginous yeasts from dairy waste
}

\author{
CN Khobragade ${ }^{1}$, Shweta R Gophane ${ }^{1}$, Vinod B Banasavade ${ }^{1}$ and NB Marathe ${ }^{2}$
}

Received: 07 November 2019 / Accepted: 04 April 2020 / Published online: 12 July 2020

(C) Indian Dairy Association (India) 2020

\begin{abstract}
Microbial lipids (biodiesel) can be produced by oleaginous yeasts via converting carbohydrates into lipids under certain conditions. Sudan black 'B' staining technique was used to screen cellular lipid accumulation. Extraction of the bacterial lipids was carried out by Bligh and Dyer method and fatty acid methyl esters were analysed by GC. Primary screening of sample resulted in 22 isolates, out of which 6 isolates were found to generate lipid. After detection of good oleaginous lipid productivity, one best yielding isolate was preferred for upward spring. Secondary selection of potential isolate was based on the production in addition to parameters like $\mathrm{pH}$ and nutritional requirements. Lipid from selected isolate CNK 1 was subjected to determination of saponification value and PUFA screening. The isolate CNK1 was identified as Zygrosaccharomyces rouxii by FAME analysis. The above results were promising hence it has importance for supplementary development of the yeast isolate Zygrosaccharomyces rouxii as another source of lipid for biodiesel production.
\end{abstract}

Keywords: FAME-GC, PUFA, Oleaginous Yeast; Sudan Black $\mathrm{B}$, Zygrosaccharomyces rouxii,

${ }^{1}$ School of Life Sciences, Swami Ramanand Teerth Marathwada University, Nanded-431606 India

${ }^{2}$ Karnataka Milk Federation (KMF), Karnataka, India

Shweta R Gophane ( $\square)$

School of Life Sciences, Swami Ramanand Teerth Marathwada University, Nanded-431606 India

Email: shweta.gophane@gmail.com;Telephone: +919405544465

\section{Introduction}

Lipid is the brief and storage form of energy require for metabolism. However, it is not only the energy contributor for an organism but also the imperative building block. Phospholipids are one of the most important compounds of biological membrane. Under certain situation, some microorganisms convert carbohydrate, hydrocarbon and normal lipid into lipids within the cells. Prior studies suggested that oleaginous microorganisms are mainly bacteria, yeast, filamentous fungi and microalgae. It was also reported that the lipid content in microalgae, yeasts and filamentous fungi was higher (70\%-90\%) than that in bacteria (20\%-50\%) (Yi Cao et al. 2012). Lipid accumulation in oleaginous yeasts and molds has been demonstrated to occur when a nutrient in the medium (i.e. the nitrogen or the phosphorus source) becomes limited and the carbon source is present in excess. Nitrogen limitation is the most efficient condition for inducing lipogenesis. In oleaginous species, the excess carbon preferentially channelled toward lipid synthesis, leading to the accumulation of TAG within intracellular lipid bodies. The ability to accumulate high amounts of lipid depends mostly on the regulation of the biosynthetic pathway and the supply of the precursors (i.e. acetyl-CoA, malonyl-CoA, and glycerol-3phosphate) and the cofactor NADPH (Ratledge and Wynn 2002). The most deeply investigated oleaginous yeasts belong to the genera Yarrowia, Candida, Rhodotorula, Rhodosporodium, Cryptococcus, and Lypomyces. Exploitation of oleaginous filamentous fungi for biodiesel production has a more recent history with few exceptions, derives from studies focused to poly-unsaturated fatty acid production (PUFA), such as arachidonic acid and linolenic acid (Rossi et al. 2011). Among heterotrophic microorganisms, oleaginous fungi, including both molds and yeasts, are increasingly been reported as good triglycerides (TAG) producers (Certik et al. 1999). The impact of drastic increase in energy consumption, concerns about green house gas (GHG) emissions, the rising price of fossil fuels and the projected decrease and insecurity of their existence in the fossil fuel reserves, have led to high attention microbial lipid production for bio-diesel production, so present work is undertaken. 


\section{Materials and Methods}

\section{Soil sample collection}

Soil sample were collected from district milk collection centre, Nanded (industry waste area).

\section{Enrichment of microorganism}

$1 \mathrm{gm}$ soil sample was added in to $250 \mathrm{ml}$ flask containing $100 \mathrm{ml}$ sterile enrichment medium (composition (g/L): Glucose-100, Yeast extract-1, $\mathrm{NH}_{4} \mathrm{Cl}-1, \mathrm{KH}_{2} \mathrm{PO}_{4}-2, \mathrm{MgSO}_{4}-0.75, \mathrm{CaCl}_{2}-0.05, \mathrm{ZnSO}_{4}-$ $0.01, \mathrm{FeCl}_{3}-0.01$ and $\left.\mathrm{Na}_{2} \mathrm{HPO}_{4}-1\right)$. This mixture was cultured at 28 C, $120 \mathrm{rpm}$ for $48 \mathrm{hrs}$. Serially diluted soil samples were spread on sterile enrichment medium plate. Then plates were kept in incubator at $28 \mathrm{C}$ for 1-4 days. After incubation each separate colonies were picked and stored on same medium agar slant for further study. Screening was done by lipid staining (Burdan K 1946).

\section{PUFA producers screening $/ \mathrm{H}_{2} \mathrm{O}_{2}$ plate assay method}

PUFA producers screening was done as per the method mentioned by (Ashwini et al. 2012). YPD medium containing (1mM) $\mathrm{NaN}_{3}$ were prepared and sterilized and isolated strains were spread on plates. After spreading sterile filter paper disc $(5 \mathrm{~mm})$ were placed on medium. $10 \mu$ l of $\mathrm{H}_{2} \mathrm{O}_{2}$ solution having different concentration $(0.5 \%$ and $1 \%)$ were added on separate filter paper disc on the plate. Plates were incubated at $28^{\circ} \mathrm{C}$ for $24-48 \mathrm{hrs}$.

\section{FAME analysis}

The fatty acids were extracted by a procedure which consists of saponification in sodium hydroxide/methanol solution followed by derivatization with hydrochloric acid/methanol solution to give the respective fatty acid methyl esters (FAMEs). The FAMEs are then extracted from the aqueous phase by the use of an organic solvent and the resulting extract was analysed by GC. FAMEs are more volatile than their respective fatty acids and therefore more suitable to GC analysis. The Sherlock software automates all analytical operations and uses a sophisticated pattern recognition algorithm to match the unknown FAME profile to the stored library entries for identification (Anju et al. 2011).

\section{Production and extraction of lipid}

$100 \mathrm{ml}$ fermentation medium were prepared and sterilized at $121 \mathrm{C}$ for 15 mins and cultures of positive screened organism were inoculated into fermentation medium. Flasks were incubated at $28 \mathrm{C}, 120 \mathrm{rpm}$ for 6 days. These set were used to determine biomass, dry weight and lipid content. Extraction of lipid was done by (Bligh and dyer 1959) method. $100 \mathrm{ml}$ cultured medium were centrifuged at $5000 \mathrm{~g}$ for $10 \mathrm{mins}$. Supernatant was discarded, pellet was washed with sterile distilled water, dried and weight was taken. $15 \mathrm{ml}$ of $4 \mathrm{M} \mathrm{HCl}$ was added and kept at room temperature for $30 \mathrm{mins}$. This mixture was kept in freezer $(-20 \mathrm{C})$ for 20 minutes and immediately transferred to boiling water bath for $10 \mathrm{~min} .30 \mathrm{ml}$ chloroform : methanol mixture was added and centrifuged at $5000 \mathrm{~g}$ for $10 \mathrm{~min}$. Lower layer chloroform contained lipid were collected and used for further study.

\section{Estimation of lipid}

Estimation of total lipid was carried out by (Barnes and blackstock 1973) method.

\section{Production media optimization study}

\section{Effect of pH on biomass lipid production}

$100 \mathrm{ml}$ fermentation medium of different $\mathrm{pH}(4.5,5.5,6.5,7.5 \& 8.5)$ were prepared. Isolated strains were inoculated into each different $\mathrm{pH}$ medium and incubated at $28 \mathrm{C}, 120 \mathrm{rpm}$ for 6 days for lipid production. After incubation the biomass and lipid content were checked in each $\mathrm{pH}$ medium. The lipid content was determined by lipid estimation. The optimum $\mathrm{pH}$ medium suitable for isolated strain was determined.

\section{Effect of carbon source on biomass and lipid production}

$100 \mathrm{ml}$ of fermentation medium of different carbon source (glucose, sucrose, lactose, maltose, and fructose) were prepared. Isolated strains were inoculated into each different carbon source medium and incubated at $28 \mathrm{C}, 120 \mathrm{rpm}$ for 6 days for lipid production. Biomass and lipid content were checked in each carbon source medium. The high lipid content was determined by lipid estimation. The optimum carbon source medium suitable for isolated strain was determined.

\section{Effect of nitrogen source on biomass and lipid production}

$100 \mathrm{ml}$ fermentation medium of different nitrogen source (organic nitrogen source: yeast extract, peptone and inorganic nitrogen source: $\left.\mathrm{NH}_{4} \mathrm{Cl}, \mathrm{KNO}_{3}\left(\mathrm{NH}_{4}\right)_{2} \mathrm{SO}_{4}, \mathrm{NH}_{4} \mathrm{NO}_{3}, \mathrm{NaNO}_{3}\right)$. Isolated strains were inoculated into each different nitrogen source medium and incubated at 28 C, $120 \mathrm{rpm}$ for 6 days for lipid production. After incubation the biomass, lipid content was checked in each nitrogen source medium. The high lipid content was determined by lipid estimation and the optimum nitrogen source medium suitable for isolated strain was determined.

\section{Saponification value}

Saponification value was determined by the methods of Association of Official Analytical Chemists (1990). 4-5 gm lipid sample was added in to flask and $50 \mathrm{ml}$ alcoholic $\mathrm{KOH}$ was added. Blank was prepared by taking only $50 \mathrm{ml}$ alcoholic KOH. Air condenser was connected to the flask and reaction mixture was boiled for $60 \mathrm{~min}$. $1 \mathrm{ml}$ phenolphthalein indicator was added and 
reaction mixture was titrated against $0.1 \mathrm{~N} \mathrm{HCl} \mathrm{up} \mathrm{to} \mathrm{pink} \mathrm{colour}$ disappears.

\section{Statistical analysis}

All the incubations were performed at triplicates and the data was analysed using MS-Excel and the result were expressed as the mean $\pm \mathrm{SD}$.

\section{Results and Discussion}

Soil sample were collected from district milk collection centre, Nanded (industry waste area) and successfully used for isolation of lipid producers. Total 43 isolates were obtained on nitrogen limited medium. Out of these isolates, 22 isolates were recovered and used for screening of lipid producers. Twenty two isolates preliminary screened trough Sudan black B staining method and six isolates showed lipid producers (Figure 1). Extraction of lipid was done by using advanced Bligh and dyer method. Amount of lipid produced in test sample was calculated by interpolating optical density of test sample on standard lipid curve and the estimated concentration of lipid was found to be $0.098 \mathrm{gm} / 100 \mathrm{ml}$. The Microbial Identification System (MIDI) for fatty acid methyl ester (FAME) analysis is a standard method for identification of microorganisms (Schutter et al. 2000). Whole cell fatty acids are converted to methyl esters and analysed by gas chromatography. The fatty acid composition of the unknown is compared to a library of known organisms in order to find the closest match. The list of the fatty acids composition like straight Chain fatty acids $21.84 \%$, branched chain fatty acid $25.50 \%$, Mono Unsaturated Fatty Acid $12.68 \%$ and oleic acid (C18:1w9c) 38.36 $\%$ was given clearly according to the GC report. The FAME GC analysis data obtained (Table 1) is more descriptive and elaborative. Our experimental data matches and establish the similar result mentioned in the report of MIDI Sherlock software databases and the similarity was matched with organism Zygosaccharomyces rouxii with selected ion monitoring (SIM) index 0.49 (Figure 2). Based on morphological characteristics and FAME-GC analysis it was identified as Zygosaccharomyces rouxii. Ravikumar et al. (2012) worked on biodiesel production from oleaginous fungi which involves the mixture of fatty acyl methyl/ethyl esters, produced from transesterification of neutral lipids. In similar way, Mrinal et al. (2011) has studied the comparative lipid profiling of endophytic fungi by FAME analysis techniques. Gao et al. (2010) also studied the screening, fermentation and optimisation of microbial lipid producing molds.

\section{Production media optimization study}

\section{Effect pH on biomass and lipid production}

Effect of $\mathrm{pH}$ on biomass and lipid production were studied, the strains was incubated at varying $\mathrm{pH}$ (4.5 to 8.5). Outcomes of the stated reports (Figure 3 ) shows increase in the biomass as well as lipid yield with respect to the increase in $\mathrm{p}^{\mathrm{H}}$ value but the relevant increase was maximum at 7.5 and shows minimum at 8.5 $\mathrm{p}^{\mathrm{H}}$ respectively.

\section{Effect of carbon source on biomass and lipid production}

Effect of carbon source on biomass and lipid production was studied; different carbon sources were used in the production medium (Figure 4). The result showed lactose, maltose, glucose, sucrose and fructose were suitable carbon source for the growth and lipid accumulation of the isolated strain among which glucose is the most suitable. The lipid production was $0.092 \mathrm{~g} / 100 \mathrm{ml}$.

\section{Effect of Nitrogen source on biomass and lipid production-}

The effect of nitrogen source on biomass and lipid production was shown in (Figure 5). Organic nitrogen source (yeast extract and peptone) whereas inorganic $\left(\mathrm{KNO}_{3},\left(\mathrm{NH}_{4}\right)_{2} \mathrm{SO}_{2}, \mathrm{NH}_{4} \mathrm{NO}_{3}\right.$, $\mathrm{NH}_{4} \mathrm{Cl}$ and $\mathrm{NaNO}_{3}$ ) were used. In the organic nitrogen source yeast extract is the suitable and lipid production was $0.098 \mathrm{~g} / 100$ ml. Similarly, $\mathrm{NH}_{4} \mathrm{Cl}$ is the most suitable inorganic nitrogen source; lipid production was found to be $0.098 \mathrm{~g} / 100 \mathrm{ml}$.

\section{PUFA screening}

As shown in (Figure 6), yeast cells were grown in presence of $\mathrm{H}_{2} \mathrm{O}_{2}$ to check membrane shielding effect of PUFA. PUFAs are the most vulnerable to oxygen and ROS. To confirm this, growth of yeasts in presence of $\mathrm{H}_{2} \mathrm{O}_{2}$ was in reality mainly due to presence of PUFA; $\mathrm{NaN}_{3}$ was added into the media which is a very powerful inhibitor of catalase. If microorganism is producing catalase enzyme, $\mathrm{NaN}_{3}$ inhibits catalase enzyme. Out of selected 6 strains, strain of Zygosaccharomyces rouxii has given false positive results at all $\mathrm{H}_{2} \mathrm{O}_{2}$ concentrations used during plate assay method.

Table 1 FAME GC analysis

\begin{tabular}{llllllc}
\hline RT & Response & Ar/Ht & RFact & ECL & Peak Name & Percent $(\%)$ \\
\hline 1.000 & 24951 & 0.100 & 1.000 & 1.000 & Straight & 21.84 \\
2.000 & 3865 & 0.100 & 1.000 & 2.000 & Branched & 25.50 \\
3.000 & 5564 & 0.100 & 1.000 & 3.000 & Hydroxy & 1.62 \\
5.000 & 2485 & 0.100 & 1.000 & 5.000 & MUFA & 12.68 \\
9.000 & 168 & 0.100 & 1.000 & 9.000 & $18: 1$ w9 & 38.36 \\
12.000 & 353 & 0.100 & & 12.000 & Other & - \\
\hline
\end{tabular}


Fig. 1 Sudan black B staining

Fig. 2 FAME-GC Analysis
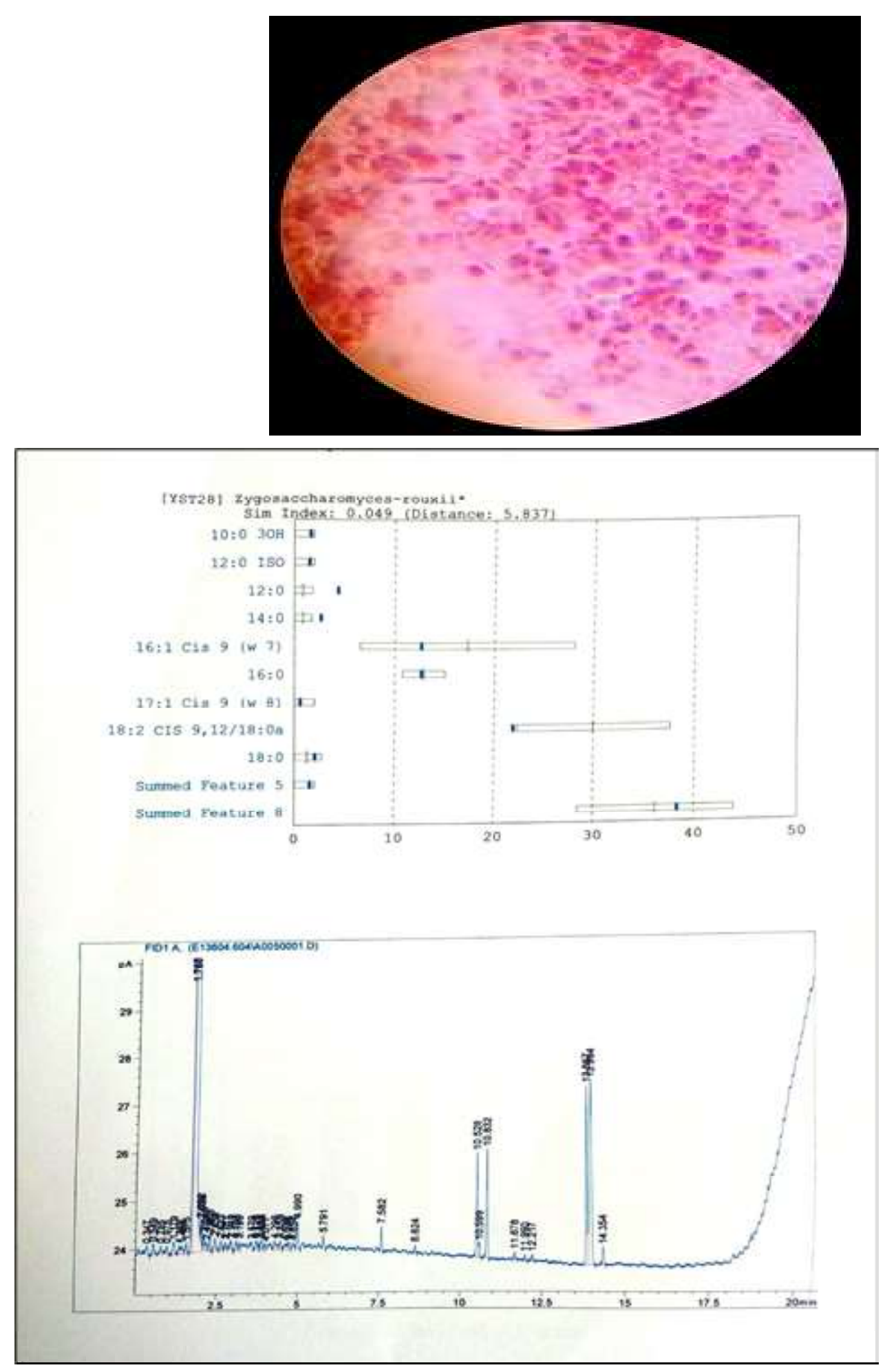

\section{Saponification value}

Saponification value for Zygrosaccharomyces rouxii has calculated 1122. Saponification value is one of the chemical properties of biodiesel which contribute to fatty acid profile. Saponification value indicates amount of triacylglycerol present in total lipid. Since, the proportion of neutral lipid is the major component in total lipid content of Zygrosaccharomyces rouxii capable of yielding high amount of lipid biomass. The basic mechanism of lipid accumulation in microorganisms has been well studied. It was observed that when the culture medium lacks the nitrogen source, the isocitric dehydrogenase (ICDH) get 


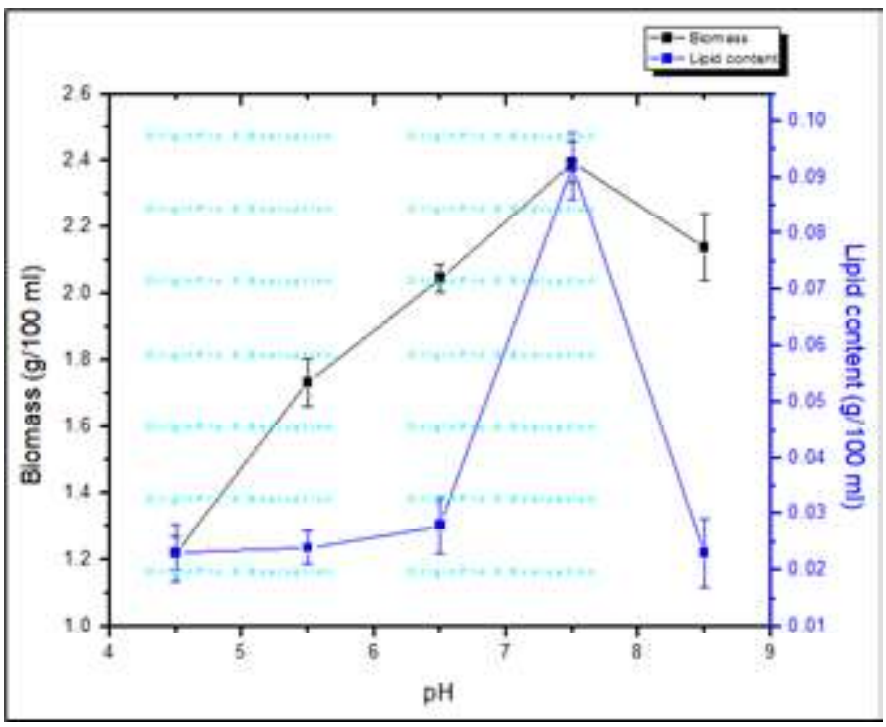

Fig. 3 Effect of $\mathrm{pH}$ on biomass and lipid production

Fig. 5 Effect of Nitrogen source on biomass and lipid production

Fig. $6 \mathrm{H}_{2} \mathrm{O}_{2}$ Plate assay method suppressed, therefore the tricarboxylic acid (TCA) was blocked.
Extra carbon source was transformed to triglyceride (TAG) by a series of enzymes like the citric acid lytic enzyme, the malic acid

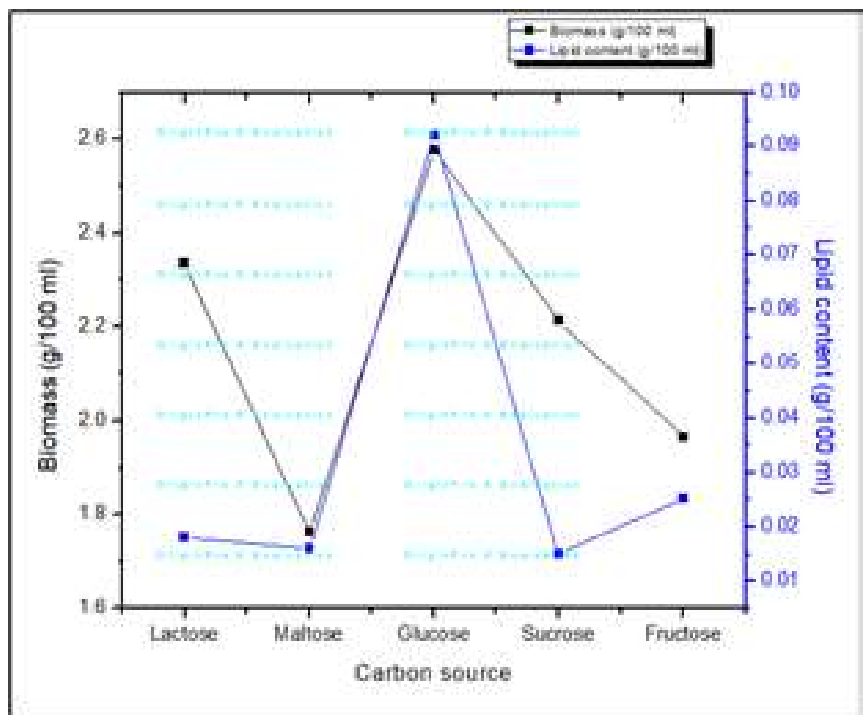

Fig. 4 Effect of carbon source on biomass and lipid production
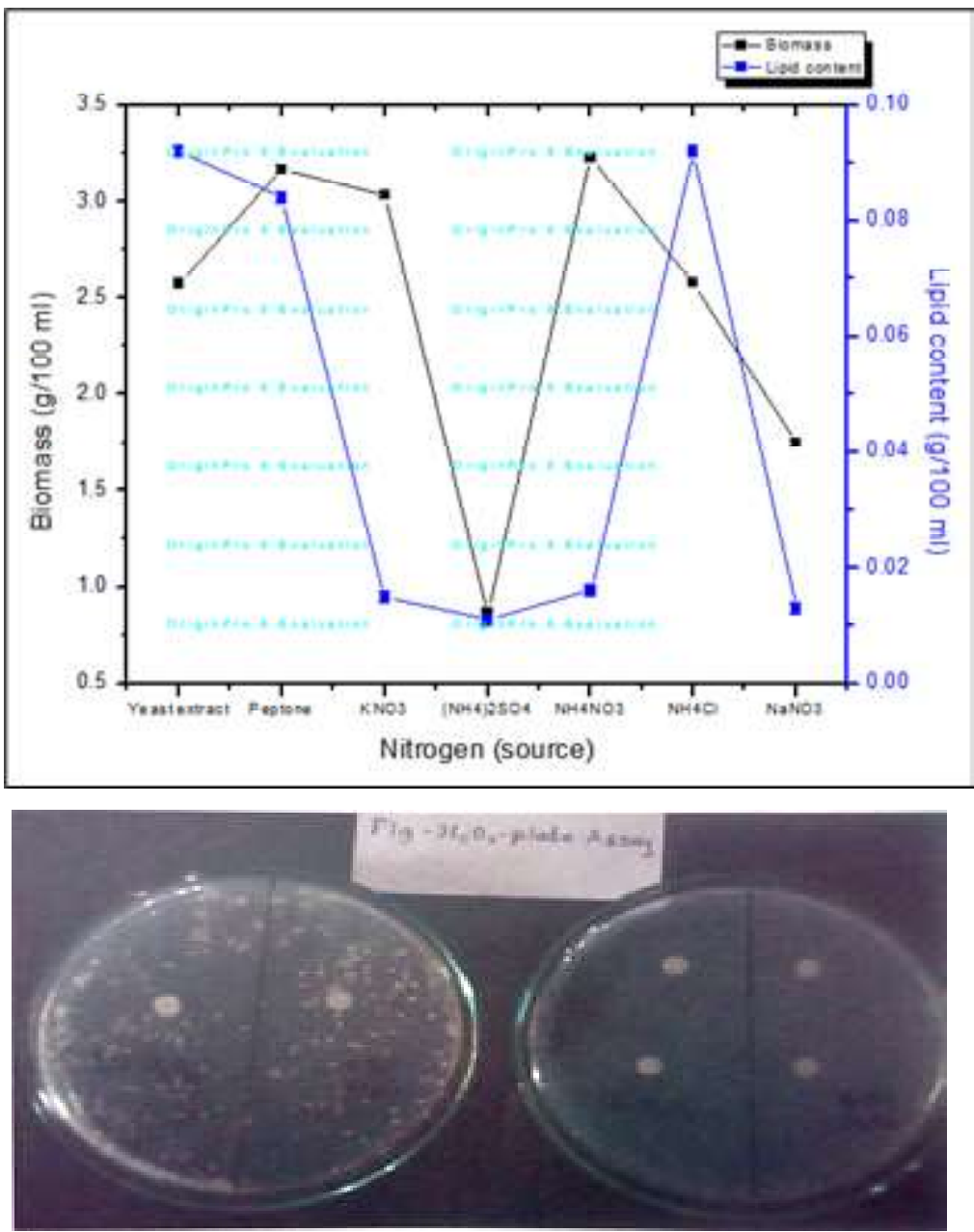

enzyme, the fatty acid synzyme, thus completed the fat accumulation. When the culture medium contains sugar but low nitrogen, lipid accumulates in order to isolate oleaginous 
microorganism, Sudan Black B staining is usually used to determine lipid content. However, this method roughly indicates the presence of microbial lipids.

\section{Conclusions}

A methodical study to search the soil sources for eukaryotes, producing lipid was performed. The study involved isolation and screening of PUFA (poly unsaturated fatty acid) producing oleaginous yeast, identification of the yeast with fatty acid profile by FAME-GC, physico-chemical parameters, optimization and saponification value from dairy waste soil sample. Primary screening of sample resulted in 22 isolates. Among them 6 isolates were found to generate lipid. Based on lipid productivity, the potent isolate CNK1 was selected and identified as Zygrosaccharomyces rouxii by FAME analysis. The lipid yield of Zygrosaccharomyces rouxii was found $0.098 \mathrm{gm} / 100 \mathrm{ml}$ in optimized media and at optimized condition. Zygrosaccharomyces rouxii has shown saponification value 1122 . In addition to that Zygrosaccharomyces rouxii is good PUFA producing and with high percent oleic acid side chain which are the best characteristics of oleaginous yeast used for biodiesel production. The above results are promising but still there is a need for protein sequencing, gene identification and other bioinformatics parameters study for further establishment of value of the yeast isolate Zygrosaccharomyces rouxii.

\section{Acknowledgments}

The authors are thankful to the Director school of life sciences, Swami Ramanand Teerth Marathwada University, Nanded (MS), India for their constant encouragement, help and support for extending necessary facilities.

\section{References}

Anju R, Navya, Aruna, Surrjit K (2011) Comparative study of FAME and sequence analysis for identification of Bacteria. Biotechnol Bioinf Bioeng 1: 319-323

AOAC (1990) Official Methods of Analysis of the Association of Official Analytical Chemists, 15th ed.; AOAC: Washington, DC; 955-972
Ashwini T, Uday Annapure (2012) Novel, simplified and rapid method for screening and isolation of polyunsaturated fatty acids producing marine bacteria. Biotechnology Research International 2012. https:/ /doi.org/10.1155/2012/542721

Barnes H, Blackstock J (1973) Estimation of lipids in marine animals and tissues: Detailed investigation of the sulphophosphovanillin method for 'total' lipids. J Exp Mar Biol Ecol 12: 103-118

Bligh E, Dyer W (1959) A rapid method of total lipid extraction and purification. Canadian J Physiol Pharmacol 37: 911-917

Burdon K (1946) Fatty material in bacteria and fungi revealed by staining dried, fixed slide preparations. J Bacteriol 52: 665-78.

Certik M, Megova J, Horenitzky R (1999) Effect of nitrogen sources on the activities of lipogenic enzymes in oleaginous fungus Cunninghamella echinulata. J General Appl Microbiol 45: 289-293

Gao D, Zeng J, Zheng Y, Yu X, Chen S (2013) Microbial lipid production from xylose by Mortierella isabellina. Bioresour. Technol 133: 315321

Ravikumar K, Dakshayini J, Girisha S (2012) Biodiesel production from oleaginous fungi. Int J Life Sci 6. doi: https://doi.org/10.3126/ ijls.v6i1.5721

Rossi M, Buzzini P, Cordisco L, Amaretti A, Sala M, Raimondi S, Ponzoni C, Pagnoni M, Matteuzzi D (2009) Growth, lipid accumulation, and fatty acid composition in obligate psychrophilic, facultative psychrophilic, and mesophilic yeasts. FEMS Microbiol Ecol 69: 363-372

Maiti M, Dey P, Banerjee J (2011). Comparative lipid profiling of two endophytic fungal isolates- Colletotrichum sp. and Alternaria $s p$. having potential utilities as biodiesel feedstock. Biores Technol 102: 5815-5823

Ratledge C, Wynn J (2002) The biochemistry and molecular biology of lipid accumulation in oleaginous micro-organisms. Advances Appl Microbiol 51:1-44

Schutter M, Dick R (2000) Comparison of fatty acid methyl ester (FAME) methods for characterizing microbial communities. Soil Sci Soc Am J 64: 1659-1668

Shi Lin Li, Qiang Lin, Xin Ran Li, Hui Xu, Yun Xi Yang, Dai Rong Qiao, Yi Cao (2012) Biodiversity of the oleaginous microorganisms in Tibetan plateau. Brazilian J Microbiol 43: 627-634 